\title{
The space weather station at the University of Alcala
}

\author{
Antonio Guerrero*, Consuelo Cid, Alberto García, Emilio Domínguez, Fernando Montoya, and Elena Saiz \\ Space Weather research group, Physics and Mathematics Dpt., Universidad de Alcalá, Plaza San Diego s/n, \\ 28801 Alcalá de Henares, Madrid, Spain
}

Received 31 May 2020 / Accepted 16 February 2021

\begin{abstract}
The Space Weather station at the University of Alcala (UAH-STA) is a place for instrumentation that is able to produce useful products and services even in a worst case scenario (when power grid and/or communications have been compromised), assuring the access of critical data to decision-makers and consequently, increasing the confidence to take actions. The current development consists of an antenna to monitor ionospheric disturbances through the reception of very low frequency waves and a magnetometer to indicate the geomagnetic disturbances caused by sources external to the Earth. This work shows the development of both instruments and some examples of ionospheric and geomagnetic events recorded by both of them. This project serves also as a success story of using space weather as a teaching tool due to the involvement of undergraduate students at their final stage of industrial and telecommunication engineering.
\end{abstract}

Keywords: space weather instrumentation / magnetometer / VLF antenna / resilience

\section{Introduction}

Space weather is a natural hazard being officially recognized nationally in some countries around the world, with a growing interest in others. Monitoring space weather should be a priority for our highly technology-dependent society. For risk management, resilience of the instruments that provide measurements is a mandatory feature. However, the question is: Are we aware of the resilience limits of the systems that are monitoring space weather activity? Many of the measurements used in monitoring and forecasting space weather are recorded by instruments on board spacecraft. These measurements include but are not limited to flaring activity, solar disc and coronal imaging, solar particle events, solar wind plasma parameters or interplanetary magnetic field data. Many of these measurements are only possible by going into space. Thus, one drawback is the increased vulnerability of space-based instrumentation to adverse conditions that might impact observations or even damage instruments, which, of course, might be the case during major space weather events. Ground measurements are also involved in space weather services, for example, ground magnetic field, ionospheric parameters or solar images at certain wavelengths. All space weather instruments and facilities have requirements, in terms of ground-based instruments they might be constraints related to environmental cleanliness, or even potential access difficulties in certain conditions, and these can lead to particular vulnerabilities or limitations. In some ground-based systems the

\footnotetext{
*Corressponding author: aguerrero@uah. es
}

space weather incstrumentation might be housed and operated from facilities that are not dedicated to space weather monitoring.

The Space Weather (SWE) station (STA) at the University of Alcala (UAH), UAH-STA, is conceived as a resilient system for monitoring real time space weather activity able to work in the worst situation, i.e. without dependencies on other infrastructures. The design allows sufficient information for decisionmakers even during hazardous space weather conditions. Moreover, the station has been successfully used as a lab for students where they are able to design, test and improve their knowledge. The station is operated by students in a winwin situation; it provides training for students in a real environment and their involvement facilitates the operation of the station.

At the time of writing this paper, the UAH-STA consists of two instruments: a Very Low Frequency (VLF) antenna and a magnetometer. The involvement of students in these two instruments is described in Section 2. The instruments are described in Sections 3 and 4. Section 5 describes common infrastructure and future development of the instruments. Finally, our conclusions appear in Section 6.

\section{Students involvement in space weather development. A win-win situation}

We cannot deny space weather is a discipline in growth. We are still discovering new effects of solar activity in our 
technology as well as new ways to detect and measure those effects. This situation is an excellent breeding ground for fresh minds like those of students at undergraduate level and higher. The UAH-STA is a successful story that proves the involvement of students as a win-win situation.

In this scenario, the VLF monitoring station at the University of Alcala was conceived at a very first stage as an academic project to involve graduate students in space weather and research activities. Two first-year undergraduate students showed interest in the project from the beginning, getting them involved through the years until presenting the work as final degree projects (García, 2019; Montoya, 2019). Their first work focused on getting a prototype of the instrument working and able to measure the activity of the Sun. After successfully capturing some flares, the students got quickly engaged in designing and building a system optimized for their own engineering requirements. The UAH-VLF system was separated in two parts, the antenna system (including preamplifier) and the data acquisition system. Each student was responsible for one of the parts. Nonetheless, they had to communicate to each other and work together integrating both parts as an individual system. Alberto García was responsible for the antenna design and the circuitry that preamplified the antenna signal. This work is described in Sections 3.1 and 3.2. Fernando Montoya was responsible for the development of the data acquisition unit described in Section 3.4. Their work was not exclusively focused on the systems engineering part, because they were involved also in understanding the data when the Sun was both active and quiet. They solved problems by their own and searched for answers to explain the observations. Some results were presented at the 15 th European Space Weather Week in 2018.

The UAH-MAG has a similar timeline, it started as a project to involve students in a previous prototype with a configuration of three single low-cost sensors and data acquisition system based on Arduino (an open-source electronic prototyping platform). Although this first prototype gave satisfactory results, the errors involved in the final construction were not acceptable for an operational use mainly due to error on orthogonality between the three axis (three individual axis were acquired from manufacturer and a single three-axis sensor was constructed out of them without proper construction and calibration tools). Later, the research group acquired three-axis sensor with better sensitivity and was able to obtain data with the required quality. The process of setting up such a system requires also knowledge and specialized work in order to obtain measurements useful for space weather purposes. Emilio Domínguez, a student in industrial engineering at the time had to work on different aspects of the project in order to get the results presented in his final degree project (Domínguez, 2019) summarized in Section 4. The first stage was the design and construction of connection wires for the different electronic modules that compose the whole system. These involved communication wires as well as power cables together with the provision of the proper power source for the equipment. Another stage of the project was the study of the place for the first measurements, evaluating the quality of the data and management of the data transmission channel and power supply facilities. Again, in this project, the student was involved in more than just getting a system recording the data. He had to interpret what the measurements showed and analyze and identify noise sources versus real geomagnetic variations.

Moreover, the students work did not end with these projects as they continue being involved in the interpretation of data and operation of the instruments. Monitoring the correct functioning of the instruments at early stages of developments is a must and students are actively involved in these activities. Unexpected shutdowns were successfully recovered, sometimes with the need of getting physically into the installation. Apart from increasing availability, every time the Sun was active they were analyzing the outputs and even tuning parameters in order to better capture disturbances.

The work presented in the following sections is the result of the involvement of students in hands-on development and operation of space weather instrumentation and is a good model for space weather application, particularly for modest ground-based stations, as well as for the development of future workers in the space weather discipline. Whilst space weather makes use of major space-based instrumentation developed by multinational consortia, this project demonstrates that, the field also requires smaller, accessible ground-based systems that can be run, effectively, with quite modest resources.

\section{The UAH-VLF monitoring station}

Solar flares are powerful releases of electromagnetic energy in the solar atmosphere, which are observed at all wavelengths from radio waves to gamma-rays. For flaring monitoring, major agencies rely nowadays on measurements of solar soft X-ray emission such as from the GOES spacecraft observations, although other ground-based flare monitoring activity exist, e.g. in H-alpha observations. Nevertheless, flares can also be monitored by considering the propagation of radio waves of distant VLF transmitters (Stokes, 1989; Raulin et al., 2010; Wenzel et al., 2016; George et al., 2019), which not only gives information about the flare but also about the actual impact on the Earth. When the electromagnetic energy released during solar flares reaches the Earth, it is absorbed in the upper terrestrial atmosphere and greatly enhance ionization of neutral species, including oxygen and nitrogen (Mitra, 1974). One of the effects of this ionization is the lowering of the effective height of the D-region, the lowest region of the Earth's ionosphere, affecting the propagation of VLF radio waves (3-30 kHz), which propagate using the Earth-ionosphere channel as a waveguide. When a flare occurs, the local electron density increases altering the VLF wave path, causing registered wave amplitude and phase to be disturbed too, especially on very long transmission paths (Bracewell et al., 1949).

The relationship between the phase delay perturbations and received VLF amplitude versus the peak of the X-ray flux was investigated in previous studies (McRae \& Thomson, 2004; Thomson et al., 2005; Kumar \& Kumar, 2018). A strong, almost linear correlation between the logarithm of the peak $\mathrm{X}$-ray flux and the measured phase change was found by Thomson et al. (2005). This trend allowed to give the size of the greatest solar flare on 4 November 2003, which saturated the GOES X-ray detectors. Regarding the received VLF amplitude, Thomson et al. (2005) obtained that it increases with the flare power, but not in a linear trend. On contrary, 
Kumar \& Kumar (2018) found that the solar flare-associated enhancements on both the amplitude and phase of VLF signals vary proportionally with the logarithm of the X-ray flux.

Nowadays, the UAH-VLF station consists of a VLF antenna receiver and a set of data acquisition, data storage and graph generation software. It is located at the University of Alcala in Spain $\left(40^{\circ} 30^{\prime} \mathrm{N}, 3^{\circ} 21^{\prime} \mathrm{W}\right)$ and has turned into an essential instrument for flaring monitoring in Spain. Hopefully one day it will be part of (at least) a European network of VLF antennas, possibly along with other existing facilities such as those described by Biagi et al. (2015) and Raulin et al. (2009), or in partnership with new ones dedicated to space weather purposes. The UAH-SWE group has already taken initiatives (at the International Workshop "Ionosphere at low frequency" (http://esters.obspm.fr/spip.php?article84) together with Paris observatory to form such a European network with these specific purposes.

\subsection{The antenna design}

Common types of antennas that are used in VLF frequency range are monopoles (whip) antennas, dipoles and loop antennas. As it is not possible to build antennas comparable to the size of a wavelength in this frequency range, these will all belong to the so called electrically small antennas category. Whip antennas and dipoles couple to the electric field of the incoming wave, while loops couple to the magnetic field. While whip antennas and dipoles have omnidirectional patterns in azimuth when used for vertical polarization, loops do not. This may be seen as an advantage as it allows us to monitor stations from different directions. However, it also leads to a higher level of interference as they can be picked up from any direction. On the other hand, small loop antennas provide two sharp nulls on azimuth. These can be used to attenuate interference sources when carefully placed. These reasons lead us to choose the so called Small Magnetic Loop (SML) antenna for the UAH-VLF station. This choice has previously shown to be a good practice as in the Stanford Space Weather monitor project (http://solar-center.stanford.edu/SID/).

Unregarding the orientation of the antenna, the effective height of an SML measures how well an antenna converts the receiving fields into electrical signals, so it has to be maximized. The expression for the effective height is given by equation (1):

$$
h_{\mathrm{ef}}=\frac{2 \pi N S}{\lambda}
$$

where $N$ is the number of turns, $S$ the area enclosed by the coil and $\lambda$ is the wavelength.

The length of the wire used for the loop sets a limit in the highest frequency able to record due to inductive effects. It is then necessary to optimally wind the loop to get the highest effective area possible for a given wire length. Considering the area of a regular polygon of $n$ sides enclosed by a fixed perimeter, the best approach will be a circumference, but this shape can pose a problem for the construction.

Maximizing $h_{\mathrm{ef}}$ can be done both, increasing the number of loops, $N$, and/or increasing the area enclosed by the antenna, $S$, for the same length of wire. Considering again the length of the wire as the primary limiting factor for the loop antenna, the equation for $h_{\text {ef }}$ shows a dependence with $N^{-1}$. Thus, a big single loop would be the best theoretical option, but again, a very large loop can be a problem during the manufacturing process.

Combining efficiency and easiness of construction, an octagon is a decent bet, as it only has a $6 \%$ area reduction over a circle while being easy to build. For this specific case, a total of $250 \mathrm{~m}$ of wire have been used which, when arranged in an octagon of side length $44.9 \mathrm{~cm}$, produced a total of 68 turns. This provides a total effective area of $69.22 \mathrm{~m}^{2}$ which translates into an effective height of $29 \mathrm{~mm}$ at $20 \mathrm{kHz}$.

Regarding the physical build, the structure of the antenna must be able to withstand adverse weather conditions in case it is placed outside. Electrical conducting materials should be avoided, as they would form a loop by themselves and, while plastic fits our electromagnetic requirements, it is sometimes difficult to build a structure that can support itself without deformations. This can be solved by using proper wood that can withstand outdoor conditions. From the wide range available, iroko wood was chosen to build the structure. This type of wood is commonly used in boats and doors and requires minimum maintenance. Several beams were given to a carpenter, with the instructions to build an octagon with its support structure. The resulting masterpiece is shown in Figure 1. It has to be noted that no screws or nails were used to join the different pieces of wood, just wood joinery techniques. Once the structure is complete, the loop can be wound. Using 23 American wire gauge (AWG) solid core copper wire a total of 68 turns were made. These all fit in a groove carved on the wood in the side of the loop, shown in Figure 1. Finally, once the coil is complete, in order to improve interference rejection, the exceeding length of the wires must be twisted until they are soldered to the coaxial cable.

The electrical properties of the antenna were studied with a lumped element model with a non ideal load recreating a passive filter. Modelling these components has no standard procedure and the result might always differ from real values. Taking into account resistance, capacitance and inductance parameters calculated with the help of the formulas given in Grover (2004) inductance the cut off frequency was found over $100 \mathrm{kHz}$, far from the frequencies of interest.

\subsection{The circuitry: preamplifier and power supply}

Although some kits are readily available for the preamplifier and power supply (The Stanford SuperSID is now available throughout the Society of Amateour Radio Astronomers (http://www.radio-astronomy.org/contact/SuperSID)) the design and development of these circuits can be used as an educational practice for engineering students. With this purpose in mind and taking as a reference the Atmospheric Weather Electromagnetic System for Observation, Modeling, and Education (AWESOME) receiver (Cohen et al., 2009), a prototype was built around a common personal computer (PC) sound card which would sample the signal coming out of the antenna. These sound cards usually come in a range of sampling rates, from the typical $44 \mathrm{kHz}$ to the high end $192 \mathrm{kHz}$ (due to Nyquist-Shannon Sampling Theorem, the highest frequency able to recover will be half the sampling frequency). Additionally, using a PC would satisfy several needs as the storage media, sampling device and network connection are easily available. It also provides enough computing power to compute the Fast Fourier Transform (FFT) in real time. Gaps in the recorded data are filled with $\mathrm{NaN}$ 

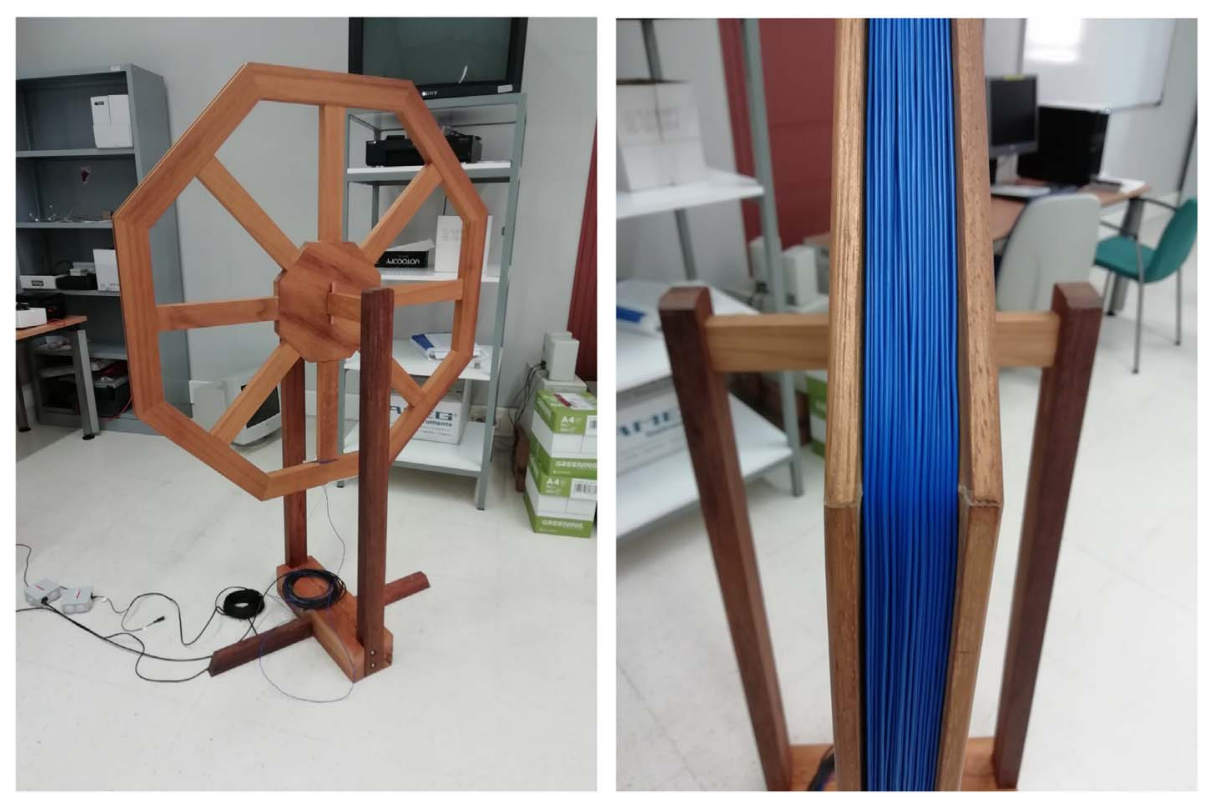

Fig. 1. Antenna structure with an octagon shape (left) and coil winding (right).

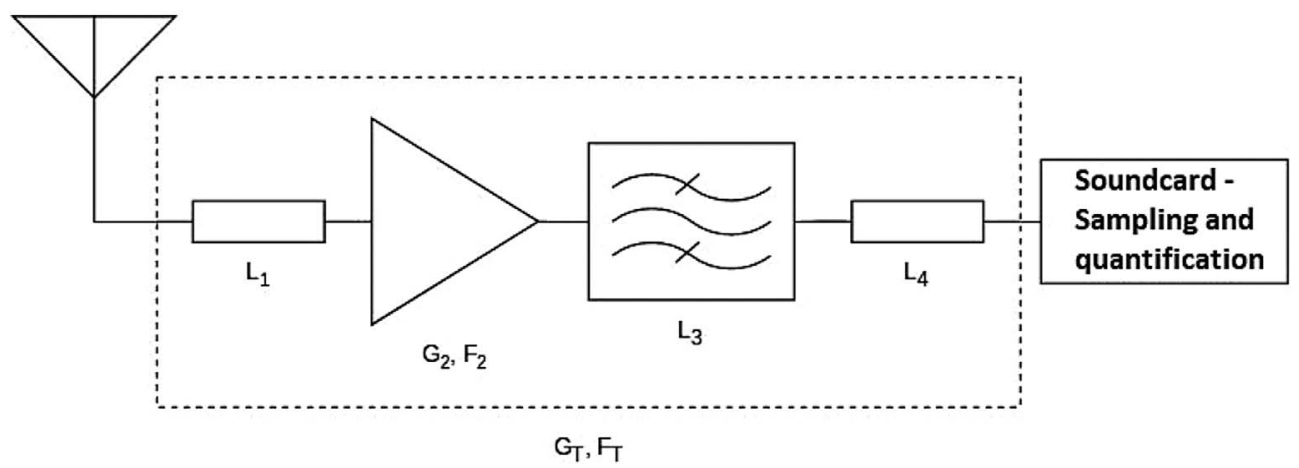

Fig. 2. Simplified block diagram of the complete system.

(Not a Number) to avoid transients when applying the digital processing algorithms and to ensure that all logs have the same number of samples.

The signal levels coming out of the loop are too weak to be used, so they need to be boosted. As the antenna is conceived to be placed far away from the computer, the signal will travel over relatively long wires. This makes amplification necessary to give the level of signal to noise ratio needed at the other end. In addition, an active filter will filter out unwanted frequencies.

The simplified block diagram for the complete system appears in Figure 2. It consists of an amplifier with noise figure F2 and gain G2, a filter with attenuation L3, and the cables that carry the signal from the antenna to the amplifier and to the computer modeled as passive purely resistive attenuators with attenuation L1 and L4 respectively. The receiver chain can be characterized with its total gain and the noise factor. Though the total gain is determined by the gain of the filter and the attenuation of the cables, the noise factor can be optimized with the arrangement of the elements by placing the element that offer gain at the beginning of the chain.

Instead of sending DC over a cable all the way up to the amplifier, it was decided to send the AC output of a transformer which also suits the selected power supply topology. The connections of the whole system are made up of $3.5 \mathrm{~mm}$ audio jacks.

The amplification uses a design in two stages. The first one is suited by the instrumentation amplifier INA128 by Texas Instruments providing an output proportional to the voltage difference between the two inputs. The instrumentation amplifier is configured using the AC-coupled design given in its datasheet using an operational amplifier (OPA2228D) as feedback to create an active filtering stage. The second stage uses also an operational amplifier and offers a low pass filter with variable gain and fixed cut off frequency.

The power supply for the amplifier is also part of the UAH-VLF design. When testing the amplifier, the current consumption was measured to be close to $20 \mathrm{~mA}$, thus a linear 
power supply suits our needs as it offers a high ripple rejection and at the same time it is easy to design and build. In addition, we are also avoiding the switching noise of switched mode power supplies. In our design, the transformer sits next to the computer and sends the low voltage AC power to the rest of the circuit (used for rectification), which is right next to the antenna and the preamplifier. The rectification circuit converting $\mathrm{AC}$ to $\mathrm{DC}$ is composed of a full bridge rectifier and a linear voltage regulator (LM317). This regulator gives up to $100 \mathrm{~mA}$ and its output voltage can be configured with two resistors.

Once the system was all set up, a computer was configured with the SuperSID software, coded by EricGibert, available at GitHub (http://github.com/ericgibert/supersid). This software controls all the sampling and logging tasks that are required to monitor the selected frequencies, and outputs the raw data to a file. Data were taken at $5 \mathrm{~s}$ sampling period and then filtered by a moving average of 13 points. The data are plotted by an adaptation by Alberto García of the SuperSID plotting code to include additional functionality which is available at GitHub (http://github.com/AlbertoGM97/sid_plot). The real time output as well as an archive are kept at http://vlf.spaceweather.es.

\subsection{From raw data to space weather monitoring}

The current UAH-VLF version uses the design shown previously and gives real time plots that can be accessed at the previously given website. This design was motivated by the results from a previous prototype that was able to capture the intense flaring activity in September 2017. This first prototype of the VLF monitoring system consisted of PVC structure and a crude preamplifier and power source implementation but demonstrated the possibilities of a monitoring station.

A prototype was ready by July 2017 at the Polytechnic school. While in the training phase, several anomalies were recorded due to the flaring activity of AR12674 in September 2017. These anomalies showed us that the UAH-VLF monitoring station was working properly, although in a noisy environment and motivated the improvement for a more robust system (shown in Fig. 1). Due to low solar activity after the new system was set up, the results in this paper belongs to the prototype. However, minor flares have been recorded by the new system. Figure 3 shows two flares happening on 6 September 2017 as recorded by GOES in soft X-ray $0.1-0.8 \mathrm{~nm}$ flux and by the amplitude of the UAH-VLF receiver when monitoring the $19.6 \mathrm{kHz}$ signal emitted by the Anthorn Ratio Station transmitter with the call sign GQD at Anthorn, UK $\left(54.911^{\circ} \mathrm{N}\right.$, $3.280^{\circ} \mathrm{W}$ ), with a time resolution of $5 \mathrm{~s}$.

While time resolution is crucial for determining when a flare event starts, has its maximum, etc., it leads to a sparse spectrum where the flare information is concentrated at the lowest frequencies. This increases the total noise bandwidth and lets high frequency interferences get through which can mask out smaller events. To solve this, a two stage digital FIR filter was designed to remove these effects, featuring a zero phase total response and side lobes below $-40 \mathrm{~dB}$ on each stage (for more information see García, 2019).

\subsection{An alternative data acquisition unit}

The UAH-VLF monitor includes also a data acquisition unit designed to substitute a personal computer and sampling device

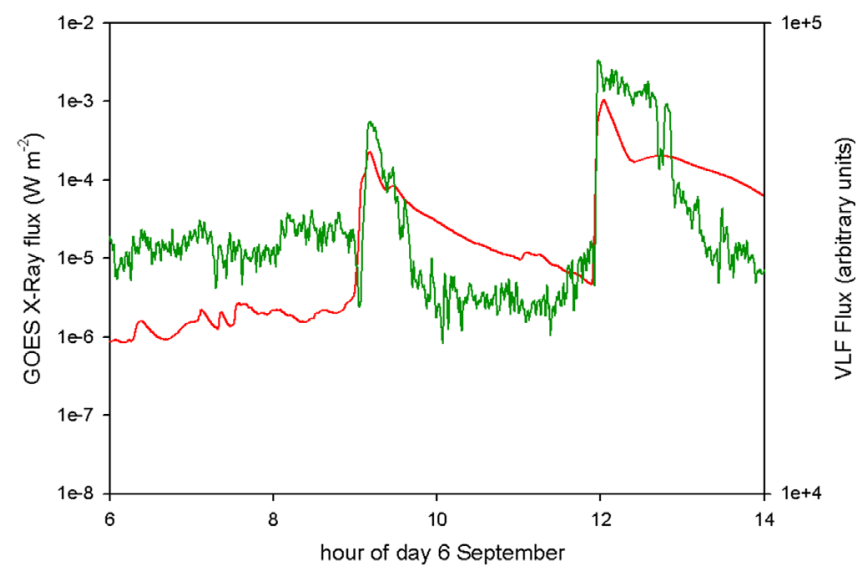

Fig. 3. Superposed plot of the GOES X-ray flux (red) and the amplitude of GQD recorded at UAH receiver (green) from 6 to 14 UT on 6 September 2017.

by the sound card. This design has been properly tested and offers also specific functionality and options in its own display. The device is planned to be installed in the next update of the UAH-VLF.

After studying different approaches from different microcontrollers available in the market, the choice was a system based on the Arduino Due board. The study took into account microcontrollers with good embeded analog to digital converters (ADC), like ST Microelectronics family and simple microcontrollers with its own external ADC designed for this kind of signal. Arduino is an open-source electronics platform based on easy-to-use hardware and software. The flexibility of its Integrated Development Environment (IDE), the accessible libraries and the hardware built to work together with it, could facilitate the work of integrating all the components of the system. About the available boards, the requirements of the project made the choice difficult because the microprocessors offered by this platform usually have low capabilities and are used for low requirements. The typical boards are Arduino UNO, with an 8-bit processor with $20 \mathrm{MHz}$, and Arduino Mega, with more input and outputs ports but with an 8-bit processor with $16 \mathrm{MHz}$ also. On the other hand, Arduino has a board based on the well-known 32-bit Cortex-M3 processor, called Arduino Due. This board implements the AT91SAM3X8E microcontroller with 32-bit bus, clock speed of $84 \mathrm{MHz}$, Direct Memory Access (DMA) hardware, 12-bits ADC up to $1 \mathrm{msps}$ (mega samples per second), and so on.

The heart and brain of the system is this Arduino board. The rest of the hardware that complement the system is an TFT Screen, a Real Time Clock (RTC), SD memory card and another microcontroller called ESP8266. The TFT screen is designed to show a menu when the system is turned on. You can select four options that is, basically, to view the signal in real time that the board is processing, view the spectrum of that signal, select 4 frequency components to store in both the SD card and in the SWE-UAH server, and the option to start logging the data of this 4 frequency components selected. Each frequency component corresponding to each receiving VLF station. The purpose of the RTC is to keep the time with an accuracy of about \pm 50 parts per million. 
To store the data, the system starts to sample the input and store the digital values in a buffer in memory. When the buffer is empty, a Fast Fourier Transform is applied to decompose the signal in its frequency components and then store the amplitude of 4 frequencies selected in other buffer. When a minute is passed, the microcontroller takes the average of the values stored in this set of time and, with this final value, the system stores the data. The data stored is a csv file in the SD card that shows in each row the 4 frequencies and its corresponding amplitudes with the timestamp at the end of the row. In addition to this, the system sends this average value through a serial communication to the other microcontroller (ESP8266) in order to upload the data to the SWE-UAH server. Once uploaded the data is publicly available for users and/or forecasters with a delay of minutes after the value is obtained.

The ESP8266 is a low-cost Wi-Fi chip that implements a TCP/IP complete stack and a processor called Tensilica Xtensa with 32 bits and $80 \mathrm{MHz}$. Although the processor is nearly the same as AT91SAM3X8E, the poor Input/Output (I/O) capability and the low memory capacity do not allow us to implement all functions on it. This chip can be programmed through Arduino IDE as well. A simple program is stored in its memory to communicate with the Arduino Due to receive the data and then upload them through a GET command (HTTP protocol) to the server. In the server there is a php web page that receives the data and saves them in a text file which can be viewed through the internet.

The screen used is a $2.8^{\prime \prime}$ SPI module based on an ILI9341 controller. This screen has also a touch panel that lies under the display. Furthermore, the PCB of the screen counts also with a SD slot and the corresponding tracks to implement the SPI protocol also with this memory hardware. For these reasons together with the existence of an Arduino Due library to handle this controller using the DMA, this choice fitted very well our purposes.

The RTC is based on the DS323 chip. This clock is used to give the timestamp of the samples in order to know when the signal is processed. The system creates in the SD card the file corresponding with the current day named according to the year, month and day.

Another specific implementation on the system, which was part of a bachelor thesis (Montoya, 2019), is the code to calculate the Fast Fourier transform. The tests to check the complexity of the algorithms for the Discrete Fourier Transform (DFT) and the FFT were also part of the work. For more details we refer the reader to the cited work. Current data recorded by the SWE-UAH station can be accessed publicly at http://sta. spaceweather.es.

\section{The magnetometer station: UAH-MAG}

The primary tools traditionally used to study solar-terrestrial relations are geomagnetic indices, which have been used to characterize geomagnetic variations due to forces that are external to the Earth. The irregular geomagnetic variations caused by solar activity are also one of the main sources responsible for disruption of technology. In consequence, geomagnetic indices are mainly produced by removing the contributions of regular variations to geomagnetic measurements on the ground.
This simplified picture sets the measurements of the geomagnetic field on the ground as a primary and an essential tool to monitor and diagnose space weather activity.

Geomagnetic observatories around the world usually need two sets of equipment to precisely measure the geomagnetic field for long periods. One of the systems is dedicated to measure the absolute magnitude of the geomagnetic field vector while the other system is dedicated to the variation (variometers). For space weather purposes the information given from variometers is more relevant at current stage of knowledge. Geomagnetic stations with variometer only equipment are much more simpler to design, install and operate. This fact has resulted in an increase of geomagnetic stations around the world in the last decades and the creation of initiatives like SuperMAG (Gjerloev, 2009) to compile most of these data. The magnetometer station UAH-MAG was born in this context (although the data are not currently uploaded to SuperMAG it could be so in the future), with the specific purpose to serve as an indicator of regional geomagnetic disturbances integrated in the global objectives of the UAH-STA given in Section 5. This section describes the development of the instrument and its current state.

\subsection{Configuration of the system}

The basic equipment for measuring geomagnetic disturbances consists of a tri-axial fluxgate sensor, an analog to digital converter and a computer to register the data. This basic equipment is enhanced with data processing techniques focused on removing environmental noise and solar regular variations in order to be left with a useful signal representing geomagnetic disturbances from sources external to the Earth.

The magnetometer sensor is a low noise $(25 \mathrm{pT} / \sqrt{\mathrm{Hz}}$ at $1 \mathrm{~Hz}$ ) and low power (3.3-3.7 V) LEMI-031 from Lemi LLC (http://www.lemisensors.com). It is also characterized by a low temperature drift $\left(<1.5 \mathrm{nT} /{ }^{\circ} \mathrm{C}\right)$. The analog signals from the three axis channels go to the 24-bits analog/digital converter ObsDAQ from MinGeo Ltd (http://www.mingeo.com) which are sampled simultaneously and then recorded by its companion computer MAGREC-4B. This equipment has previously shown to be capable of recording $1 \mathrm{~s}$ resolution with the INTERMAGNET quality standard (Pedersen \& Merenyi, 2016). The configuration and setup test of the system was also part of an academic project at the Polythecnic school (Domínguez, 2019). It is important to state that comparing with similar equipment, and collaboration between institutes with similar equipment, world-wide, is essential as it allows for event confirmation and better coverage, as well as for standardization.

All connection cables between components of the system were created for this purpose as well as power cables and power source for the components. The only exception to this was the connection from the sensor to the conditioning board (preamplifier) which was provided by the manufacturer. From the pre-amplifier to the Obs-DAQ we use a $10-\mathrm{m}$ cable with 12 wires of $0.5 \mathrm{~mm}$ section and shielded for interference. Each axis of the sensor gives a differential signal with a common virtual ground for the three of them, thus, only seven wires are needed for the signal of the three components. Two more wires are used to power the device from a regulated power supply giving a constant $3.5 \mathrm{~V}$ (recommended 3.3-3.7 V). 


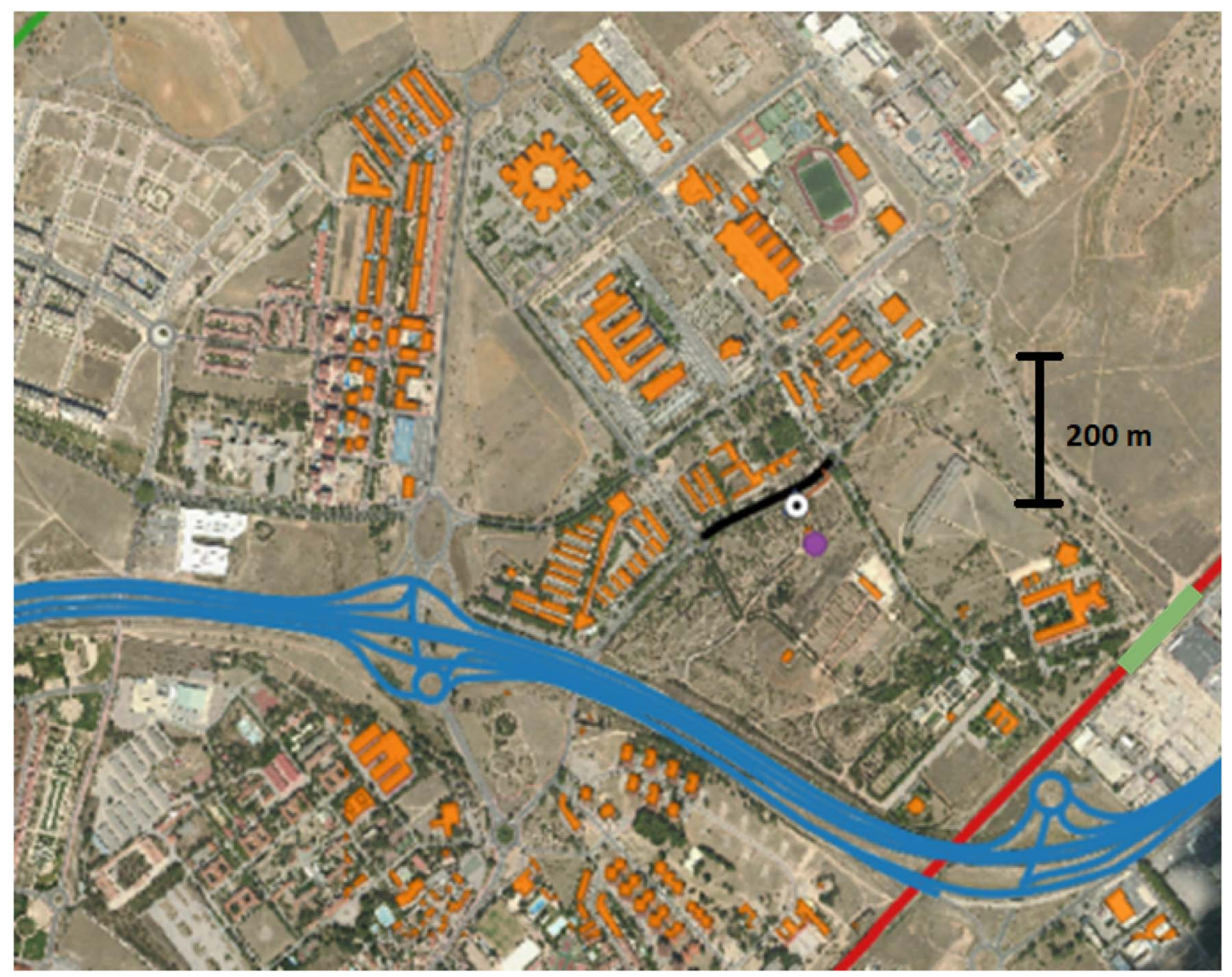

Fig. 4. Map showing the environment surrounding the station. The black dot inside a small white circle shows the location of the magnetometer test recordings. The Madrid-Barcelona highway is shown in blue, railways are marked in red and close buildings are colored in orange.

The cable is connected to the conditioning board by a single connector with 10 pins (one is unused). On the other side of the cable, the Obs-DAQ needs separated differential inputs for each of the axis. These are provided by USB type B mini connectors.

The connection between Obs-DAQ and MAGREC uses the manufacturer recommended configuration, which is the RS485 connector on the Obs-DAQ side and the COM3 on the MAGREC side. The computer (MAGREC) runs a linux system and manufacturer software already installed on it. The communication with Obs-DAQ is configured for automatic control of the bit rate. The software (MAGLIN) is configured to output the data in files following IAGA2002 format for both $1 \mathrm{~s}$ and 1 min data. The scale factor of each component is given as a first approximation as the inverse of the differential sensitivity given by the manufactured sensor $(60 \mu \mathrm{V} / \mathrm{nT})$ in volts to satisfy the units requested by the software $(\mathrm{nT} / \mathrm{V})$. Configuration parameters for the A/D conversion (input range, full-scale, sample rate, etc) are also those recommended by the manufacturer.

\subsection{Test location and first measurements}

At the time of writing this paper the UAH-MAG was already tested satisfactorily on a location of The Royal
Botanical Garden of the UAH (Real Jardín Botánico Juan Carlos I) which provided easy power supply (although no internet connection). All the results shown on this paper comes from this location. After all the test runs, the system was unmounted and ready to be placed at its definitive location with better infrastructure (see Sect. 4.3) before the COVID-19 lock-down prevented further activities here.

The Royal Botanical Garden and its surroundings are not an environmentally clean place for placing a magnetometer as we show herein, but it has other advantages which are highlighted for the main purposes of this paper and specifically for the purposes of real time space weather monitoring. The test place is located at latitude: $40^{\circ} 30^{\prime} 27.00^{\prime \prime} \mathrm{N}$ and longitude: $3^{\circ} 20^{\prime} 37.47^{\prime \prime} \mathrm{W}$. Figure 4 shows the location of the sensor and the house for the rest of the equipment with a white dot. There is a road close to the building highlighted with a black line. Even though the road is not the main road of the University campus, it is frequently used to access the surrounding faculty buildings. The blue lines mark the national highway from Madrid to Barcelona, which is one of the busiest highways in the country. The red line marks the railways which are powered by high DC voltage. The orange layer shows the location of buildings. Those closer to the magnetometer station are within the university campus. 
The data acquisition system is placed inside the house where the system is plugged in to conventional power supply. The sensor was placed outside the house using the $10 \mathrm{~m}$ cable and measurements were taken over the surface of the ground $1.5 \mathrm{~m}$ high. The sensor was inside a cork box to reduce temperature effects and the box inside another one to provide protection against rain and other problems like animals or insects.

For the purpose of this first test, the sensor was oriented with the $X$ component pointing north with the help of a compass. Orthogonality between components is assured by the manufacturer (three axis embedded together) and horizontal orientation tested with a spirit level. For space weather purposes the horizontal component of the geomagnetic field gives the most useful source of information, thus, the primary efforts must be taken to the correct orientation for horizontal level than for north orientation of the $X$ component. The reason is that orthogonality between the north component $(X)$ axis sensor and the east component $(Y)$ axis sensor is assured by construction and the horizontal component $(H)$ of the geomagnetic field does not depend on the relative orientation of these axes with real north. It is important to mention here that the configuration of the sensor outside the house does not isolate completely from temperature drifts giving important measure drifts $\left(<1.5 \mathrm{nT} /{ }^{\circ} \mathrm{C}\right.$ as given by the sensor datasheet).

It is easy to predict from the description of the sensor environment given here that undesired noise and errors will be present on the measurements. Several positions were tested, in order to reduce the noise on the components, rising the sensor from the ground, with better results at more than one meter height (for more details see Domínguez, 2019). Figure 5 is an example of the measurements on the three components of the geomagnetic field for a whole day in July 2019. Background noise is present in all components but considerably higher in the vertical $(Z)$ component. The most probable reason for this effect is the railway and their high voltage DC power. The separation is less than one kilometer and recommendations for INTERMAGNET quality measurements are given to be greater than tens of kilometres Jankowski \& Sucksdorff (1996). Similar effects have been observed and studied by other authors at systems located close to railways (e.g. Pirjola et al., 2007; Lowes, 2009; Santarelli et al., 2014; Margiono \& Yusuf, 2014).

In order to study the validity of the system for space weather purposes we compare the measurements with those given by the closest official INTERMAGNET observatory, in this case the San Pablo - Toledo Observatory, SPT, in Toledo (Spain) $137 \mathrm{~km}$ northeast. As a first approximation, we study in this work only the north component of the geomagnetic field as the best axis to indicate the state of the geospace, at least at mid latitudes. Simple comparisons by eye result in big discrepancies. The reason is that most of these first measurements took place during the summer months of 2019 with strong temperature drifts. The system did not implement the registration of the temperature so a correction for this source of error was not possible in the first place. Temperature data from an official weather station at the city of Alcala were acquired and corrections were applied to the data. Figure 6 shows the comparison between the UAH station (UAH-MAG) and SPT for 5 days of data at the end of August 2019 and the first of September, where quiet and disturbed periods took place. The first panel from the top is the minimum temperature during one hour
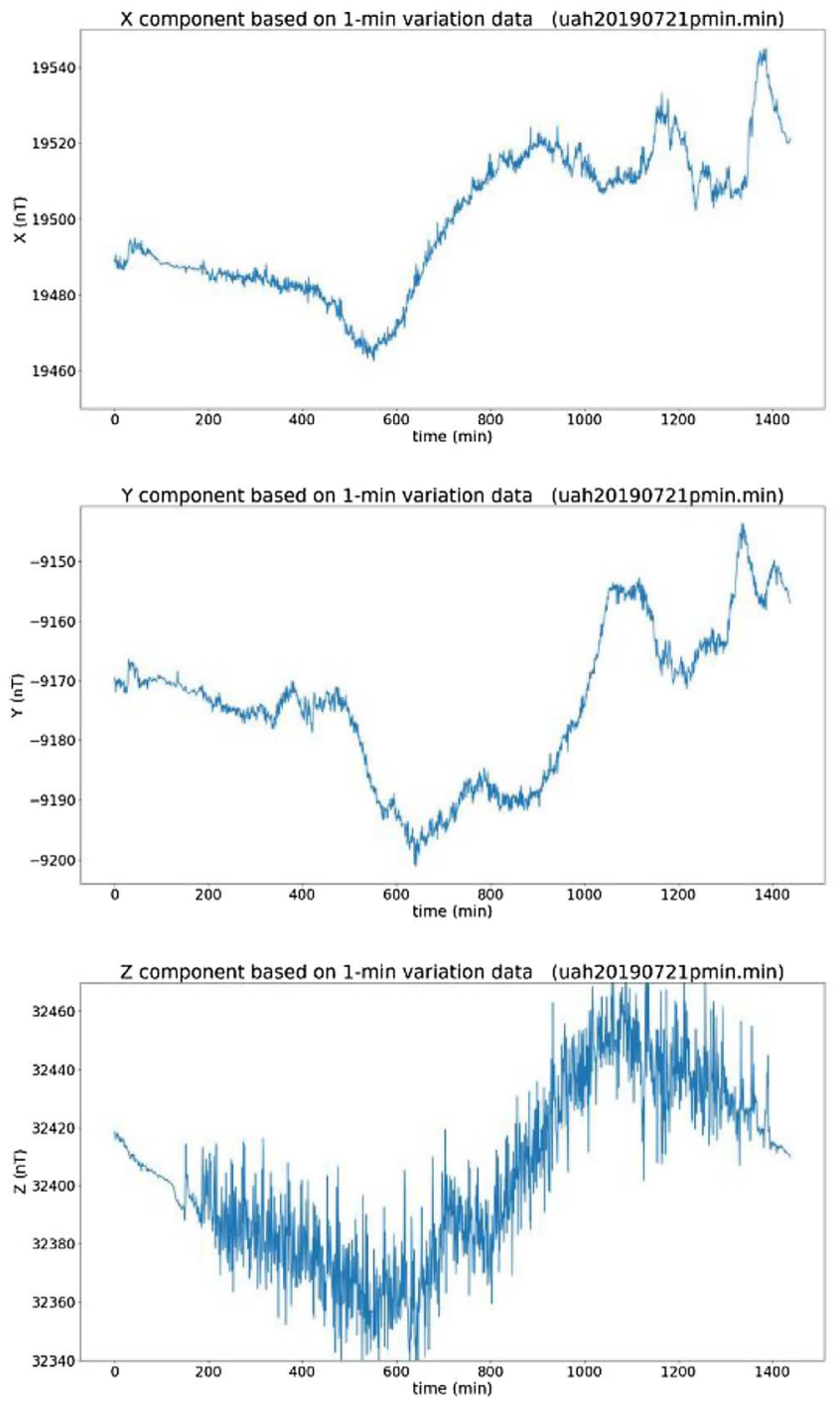

Fig. 5. An example of magnetometer recordings during a quiet day (27 July 2019). The three axes are shown from top to bottom, north $(X)$, east $(Y)$ and vertical $(Z)$ components.

interpolated for $1 \mathrm{~min}$ resolution. The second panel is the raw data measured by the UAH-MAG sensor. The third panel gives the temperature corrected data from the UAH-MAG data. The data in that panel is also corrected for the fast variation noise given above with a moving average window of $15 \mathrm{~min}$. The fourth panel shows the data measured at the official observatory as reference. The last panel is a scatter plot of the comparison with a linear regression fit to the data from the two sources. This result shows that simple corrections of this type are essential for the correct interpretation of the data. Even though the test environment and set up configuration had strong deficiencies, the corrected data show high capability of the system as a space weather tool. For example, the strongest discrepancies are shown during quiet times on the first days shown in the figure.

\subsection{Obtaining a local geomagnetic index}

Going further in the development of the station, we would like to obtain a proper indicator of geomagnetic disturbances 
Temperature at Alcala Station 3170Y

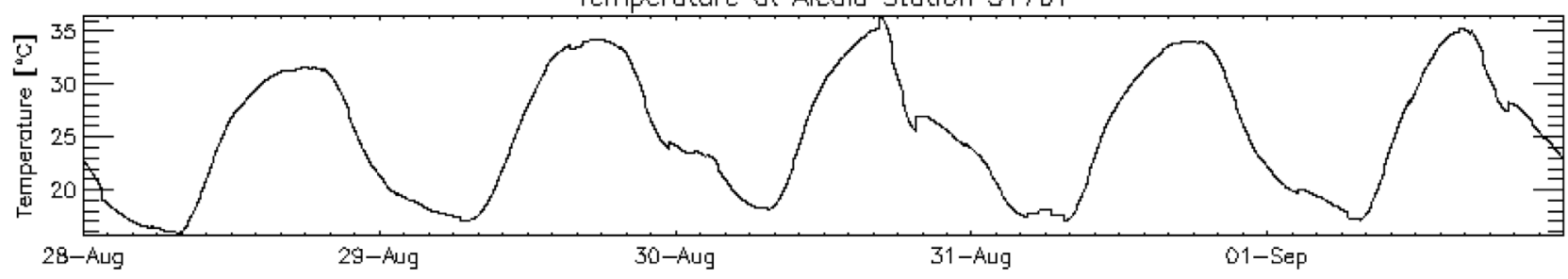

UAH-MAG row data [X component]

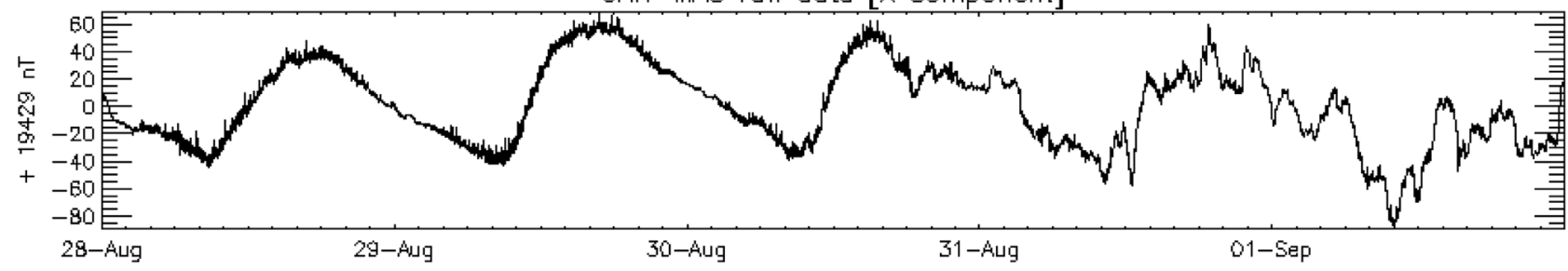

UAH-MAG corrected for temperature and noise [X component]

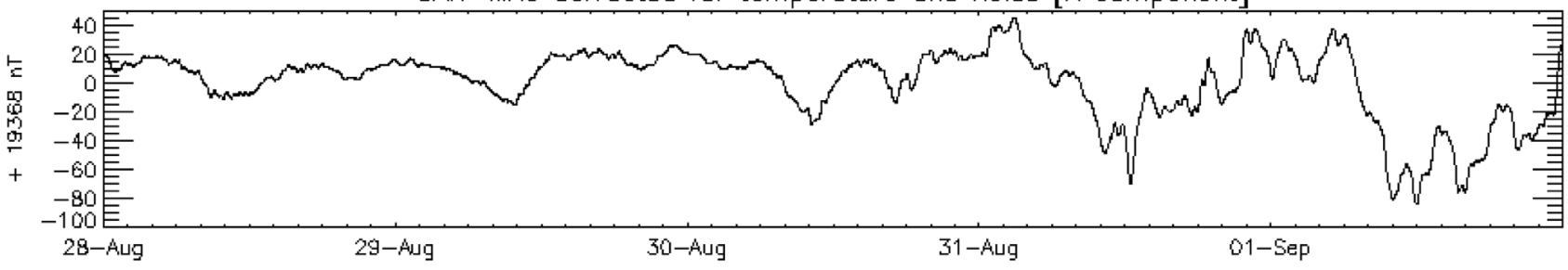

SPT observatory dato $[\mathrm{X}$ component $]$
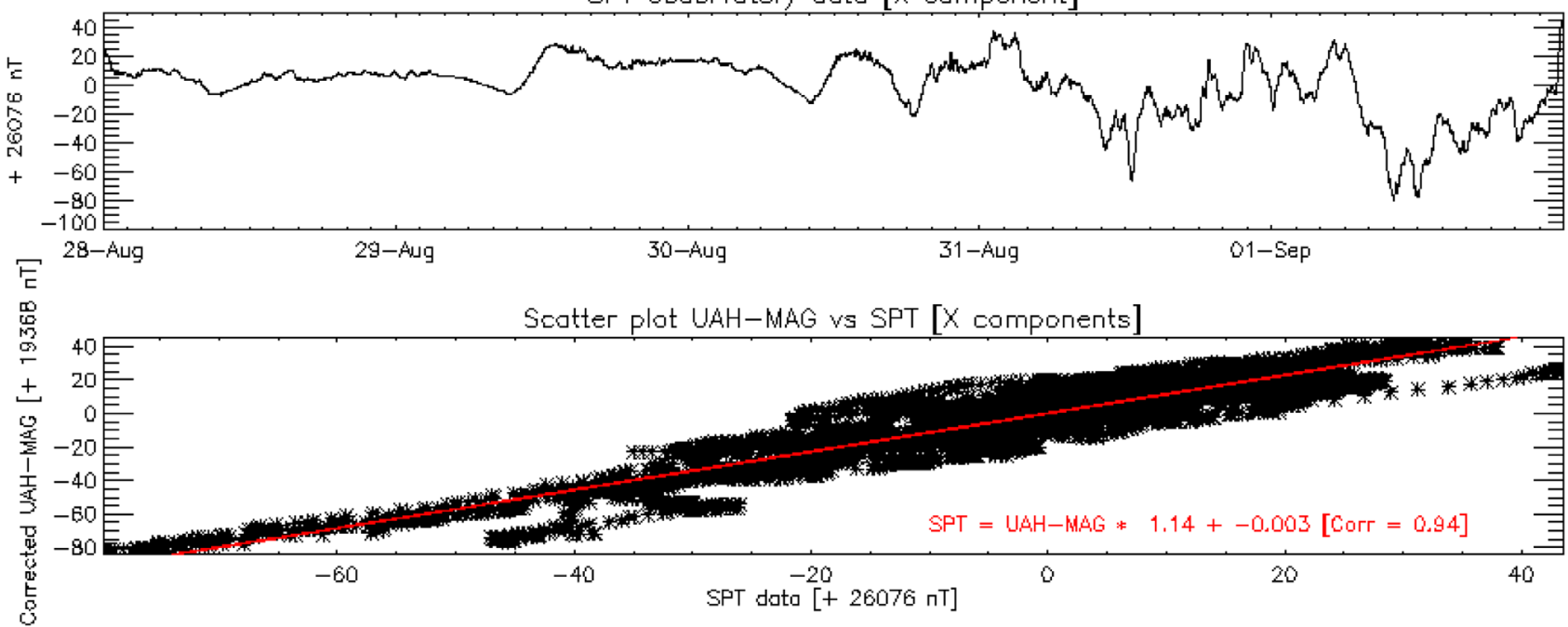

Fig. 6. Comparison of the 5 days of recordings from UAH-MAG and SPT observatory during a geomagnetic event. From top to bottom: temperature recorded at a weather station (3170Y), the recorded raw data from the magnetometer sensor, magnetometer signal corrected for temperature and noise, data from SPT observatory and scatter plot of UAH-MAG vs. SPT data with the regression fitting line and parameters obtained.

for space weather purposes. The purpose of this section is to show that even in a noisy environment the instrument can be used for space weather purposes. A local geomagnetic index is already part of the products offered by the Spanish Space Weather Service led by the SWE-UAH group, obtained from measurements of an official INTERMAGNET observatory of the Iberian Peninsula. The data are processed by a patented technique property of University of Alcala (Guerrero et al., 2016) producing a Local Disturbance index (LDi). The method is specially designed for mid latitudes, able to run in real time and with high resolution data $(1 \mathrm{~min})$. It is in a high state of development, given by an Applicability User Level of nine (AUL-9) (see Cid et al., 2019; Halford et al., 2019 for more information). The LDi procedure has been applied to UAHMAG (temperature) corrected data satisfactorily. The accomplished results are given in Figure 7 together with a comparison 

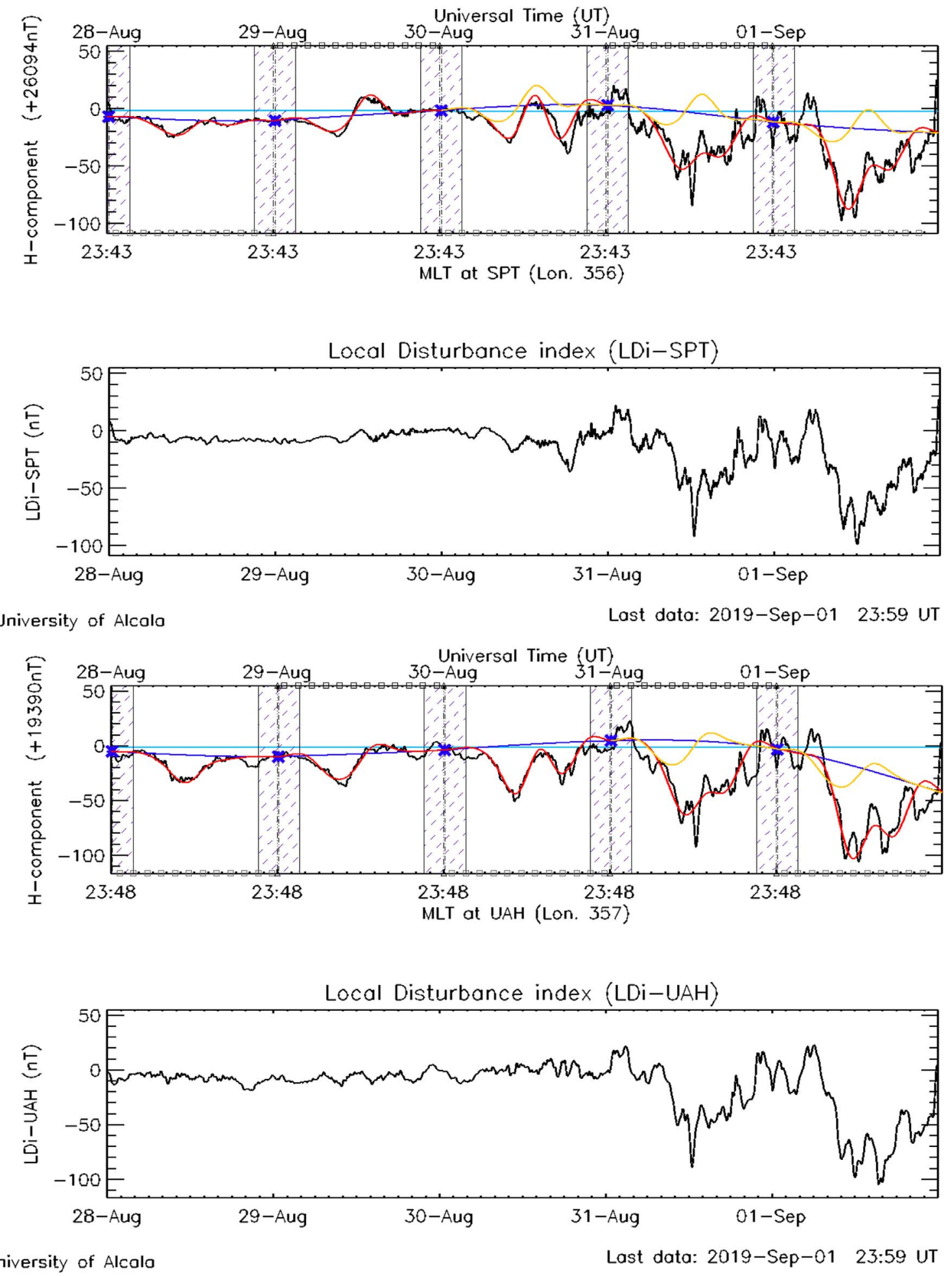

Fig. 7. Two top panels: curves involved in the procedure to obtain the Local Disturbance index (LDi) and the result for SPT data. Two bottom panels are equivalent for UAH-MAG data. Red curves are the solar regular variation fitting to the data, yellow lines are the adopted solar quiet curves, the blue line is an auxiliary curve for the calculations and the green line is the baseline considering secular variation.

with the results of applying LDi procedure to SPT data. All panels show the same time range as the Section 4.2, selected because of the presence of disturbed periods on the last 2 days of the figure (geomagnetic storm associated to fast solar wind stream coming from a coronal hole on the Sun). The top panel shows the signals involved in the LDi calculations. The shaded area marks local night time, the top axis gives UT time and the bottom axis gives magnetic local time. Since the longitude of 
the UAH-MAG location is only 4 degrees from Greenwich meridian, the difference between them is only $17 \mathrm{~min}$. The baseline taking into account secular variation is given by the light blue line close to zero on the plot. Be aware that the plot $Y$ axis has an offset given by the number in parenthesis in the same axis title. The dark blue line is an auxiliary trend line obtained from night time values and used only for other calculations in the procedure. The red line is the fitting of the model to the data (recreating the Solar Regular variation) and the yellow line is the actual data used as Solar Quiet for that day. The procedure is able to discriminate between quiet and disturbed periods and select the closest solar quiet data when disturbed period is detected. LDi data can be obtained by subtracting the baseline and solar quiet data from the measurements. The result for SPT data (LDi-SPT) is shown in the second panel (this is the reference for comparison of the index obtained from an official observatory). The third and fourth panels are the equivalent panels for the case of UAH-MAG data. The results show low discrepancies between both of them (second and fourth panels) even though UAH-MAG measurements were taken under test conditions, with no recording of the temperature of the sensor and even on a noisy environment.

\section{Common infrastructure and future development}

The UAH-STA is in its first stage of development and the next step will pursue a common infrastructure for communication and power source for the instruments following three main goals (an important point about these goals is that they are compatible with the educational purposes mentioned throughout the paper):

- To be able to keep the data safe and close (no more than a few kilometers) to the institution location providing the services.

- To have independent (from other institutions) power sources infrastructure capable to maintain operational activities.

- To obtain the lowest cost that allows proper space weather activities.

The first goal is accomplished by placing the instruments close to the University campus where the research group has their activities. The second goal needs independent power sources and redundancy if possible. In our case, power will come from solar panels that will charge a bank of batteries. Redundancy will be accomplished by a gas power supply and at last by traditional power source. In this configuration, the traditional power grid source is the last option in case other sources are not available. The solar panels are already in the definitive location for the UAH-STA within The Botanical Garden but the existing batteries still need to be tested for proper operations. When the power source is working properly, both instruments UAH-VLF and UAH-MAG, will be installed at the same location. The UAH-MAG station needs to be properly calibrated at a reference observatory.

The data transfer system relies on its own infrastructure (communication and power source) with the goal of placing the data close to the service provider (currently, the scientist). That means that every step in the communication path has to be powered by a secured and independent source. For example, mobile communication systems are not considered as they depend on systems out of the research group control. Systems that work with long range communication protocols like LoRa (Long Range) are being considered to extend the capabilities.

Furthermore, future developments of the station are focus on expanding the reception of UAH-VLF to capture the phase of the signal, which requires a system with better time keeping accuracy. The instrument will be improved also by adding the capability to receive signals in any direction. This feature can be achieved with different approaches. The antenna structure can rotate and the system will sample the signal with the angular reference in order to identify the sources. Another option is to have two antennas perpendicular to each other in order to be able to reconstruct the signals coming from any direction. In terms of software, the system can be improved updating the capabilities to record not only some frequencies (specific stations) but the whole spectrum, which requires a vast amount of data storage. A proper analysis of the data in real time could help to reduce space keeping only most relevant signals. In the case of UAH-MAG, the priority is to place the instrument in a fixed place with either sensor temperature registration or temperature control environment. Then, the instrument will undergo full calibration at an official geomagnetic observatory. The long-term goal is to be able to reproduce the instruments in other locations. The purpose is to be able to monitor ground disturbances with a higher spatial resolution than the current one and have redundancy if possible. The motivation can be found in recent results that show that some extreme geomagnetic disturbances, usually thought to be a global effect caused by the enhancement of ring current are indeed more isolated that previously known. Even the extreme local geomagnetic disturbance recorded at Colaba observatory during the Carrington event could be the result of having the right sensor (Colaba magnetometer) at the right spot and not the result of a global disturbance. For more information about this issue and how localized can be these type of disturbances (see Cid et al., 2015).

\section{Conclusions}

The (UAH-STA) is conceived as a system capable of obtaining space-weather products on its own, reducing external dependencies to other systems to zero. We have also tried to reduce the distance to the station from working offices to a minimum not to interrupt decision-maker actions when they are more important, at high risk of technological disruptions. These design pre-requisites make the station a good model easy to replicate at other locations, whilst also allowing it to be scaled up. In the development of the instrumentation we have considered low-cost materials whenever possible.

The UAH-VLF instrument started with previous designs from other institutions already known to work properly and it evolved to a new system where all hardware parts were specifically designed from scratch. This type of instrument, where VLF signals from very far stations are processed in order to obtain useful space weather information, falls into an uncommon area of science and technology joint together. This fact 
provides advantages and disadvantages. On one hand, it makes it difficult to find the best design and configuration that gives the best achievable result. The final goal must be the scientific and/or operational results that should come out of them, but sometimes this falls in a field under development, so that engineering requirements could not be easily imposed. This also opens up the door for advantages in the sense that hardware and software designs are more flexible. It is this flexibility that may result in new scientific results and sometimes unexpected uses.

The current setup of the UAH-MAG uses a tri-axial sensor with better characteristics and data acquisition system designed to record geomagnetic data and improved over a long period of years. The LDi processing technique added to the data acquired from the sensor complies the removal of a baseline and the quiet day regular variation, which allows to offer a real time indication of the local geomagnetic disturbance caused by sources external to the Earth.

The station is now on its first stage, but has shown to be able to operate in a harsh environment commonly thought not to work for these type of instruments. The current set of instruments is able to monitor solar flares (probably those with eruptive signatures) and to monitor local geomagnetic disturbances, which are the primary space weather diagnosis tool. The fruitful results shown in this paper give support to step forward in the development of an operational space weather station capable of offering exclusive products by exploiting new instrument designs and the use of new data processing techniques.

This project also proves the capability of space weather as a teaching tool. Three students from industrial and telecommunication engineering were involved in part of the tasks of this project. They developed their own designs, solved problems on their own in order to make it work, and interpreted results successfully. They participated actively in the dissemination of results in congresses or journals like this.

Acknowledgements. We acknowledge funding from the University of Alcala (project CCG2018/EXP-071) and the Spanish Ministry of Economy (MINECO) project AYA2016-80881P (including FEDER funds). We acknowledge Instituto Geográfico Nacional (IGN) for providing the ground magnetic field data from San Pablo-Toledo (SPT) observatory and the staff of SPT for their work in recording high-quality data. The editor thanks two anonymous reviewers for their assistance in evaluating this paper.

\section{References}

Biagi PF, Maggipinto T, Ermini A. 2015. The European VLF/LF radio network: current status. Acta Geod Geoph 50(1): 109-120. https://doi.org/10.1007/s40328-014-0089-x.

Bracewell RN, Straker TW, Redman RO. 1949. The study of solar flares by means of very long radio waves. Mon Notic Roy Astron Soc 109(1): 28-45. https://doi.org/10.1093/mnras/109.1.28.

Cid C, Saiz E, Guerrero A, Palacios J, Cerrato Y. 2015. A Carringtonlike geomagnetic storm observed in the 21st century. J Space Weather Space Clim 5: A16. https://doi.org/10.1051/swsc/2015017. Cid C, Guerrero A, Saiz E, Halford A, Kellerman A. 2019. Developing the LDi and LCi geomagnetic indices, an example of application of the AULs framework. Space Weather 18(1): e2019SW002171. https://doi.org/10.1029/2019SW002171.

Cohen MB, Inan US, Paschal EW. 2009. Sensitive broadband ELF/ VLF radio reception with the AWESOME instrument. IEEE Trans Geosci Remote Sens 48(1): 3-17. https://doi.org/10.1109/ TGRS.2009.2028334.

Domínguez E. 2019. Puesta en funcionamiento de un magnetómetro incluyendo estudio de ubicación, control de ruido electromagnético e influencia de la temperatura para su posterior instalación. Bachelor thesis, University of Alcala. Available at http://hdl.handle.net/10017/39514

García A. 2019. Design and construction of a VLF monitoring station for solar flare detection. Bachelor thesis, University of Alcala. Available at http://hdl.handle.net/10017/38751.

George HE, Rodger CJ, Clilverd MA, Cresswell-Moorcock K, Brundell JB, Thomson NR. 2019. Developing a nowcasting capability for X-class solar flares using VLF radiowave propagation changes. Space Weather 17(12): 1783-1799. https://doi.org/ 10.1029/2019SW002297.

Gjerloev J. 2009. A global ground-based magnetometer initiative. Eos Trans AGU 90(27): 230-231. https://doi.org/10.1029/ 2009EO270002.

Grover FW. 2004. Inductance calculations: working formulas and tables. Courier Corporation. ISBN 0-486-49577-9.

Guerrero A, Cid C, Saiz E, Palacios J, Cerrato Y. 2016. Dispositivo y procedimiento de obtencion de la perturbacion geomagnetica local a latitudes medias. Spanish Patent Office (WO2017174843A1). Available at https://patents.google.com/patent/WO2017174843A1.

Halford AJ, Kellerman AC, Garcia-Sage K, Klenzing J, Carter BA, et al. 2019. Application usability levels: a framework for tracking project product progress. J Space Weather Space Clim 9: A34. https://doi.org/10.1051/swsc/2019030.

Jankowski J, Sucksdorff C. 1996. Guide for magnetic measurements and observatory practice. International Association of Geomagnetism and Aeronomy Warsaw. http://www.iaga-aiga.org/data/ uploads/pdf/guides/iaga-guide-observatories.pdf.

Kumar A, Kumar S. 2018. Solar flare effects on D-region ionosphere using VLF measurements during low-and high-solar activity phases of solar cycle 24. Earth Planets Space 70(1): 29. https://doi.org/10.1186/s40623-018-0794-8.

Lowes F. 2009. DC railways and the magnetic fields they produce the geomagnetic context. Earth Planets Space 61(8): i-xv. https://doi.org/10.1186/BF03352944.

Margiono R, Yusuf M. 2014. On the influence of DC railway noise on variation data from tangerang geomagnetic observatories. In: 1st International Conference On Applied Electromagnetic Technology. ISBN 978-602-70279-0-9.

McRae WM, Thomson NR. 2004. Solar flare induced ionospheric D-region enhancements from VLF phase and amplitude observations. J Atmos Sol-Terr Phys 66(1): 77-87. https://doi.org/ 10.1016/j.jastp.2003.09.009.

Mitra A. 1974. Ionospheric effects of solar flares. In: Vol. 46 of Astrophysics and Space Science Library, D. Reidel Publishing Co., Dordrecht. https://doi.org/10.1007/978-94-010-2231-6.

Montoya F. 2019. Portable data acquisition and representation system for a VLF receptor. Bachelor thesis, University of Alcala. Available at http://hdl.handle.net/10017/38167.

Pedersen LW, Merenyi L. 2016. The FGE magnetometer and the INTERMAGNET 1 second standard. J Ind Geophys Union 2: 30-36. Available at https://www.space.dtu.dk/english/-/media/ Institutter/Space/English/instruments_systems_methods/3-axis_ fluxgate_magnetometer_model_fgm-fge/FGE-and-Intermagnet1-sec-standardap.ashx. 
Pirjola R, Newitt L, Boteler D, Trichtchenko L, Fernberg P, McKee L, Danskin D, Van Beek GJ. 2007. Modelling the disturbance caused by a dc-electrified railway to geomagnetic measurements. Earth Planets Space 59(8): 943-949. https://doi.org/10.1186/BF03352033.

Raulin J-P, Correia de Matos P, David P, Hadano R, Saraiva A, Correia E, Kaufmann P. 2009. The south America VLF NETwork (SAVNET): Development, installation status, first results. Geofs Int 48(3): 253-261. http://www.scielo.org.mx/pdf/geoint/v48n3/ v48n3a1.pdf.

Raulin J-P, Bertoni FCP, Gavilán HR, Guevara-Day W, Rodriguez R, et al. 2010. Solar flare detection sensitivity using the South America VLF Network (SAVNET). J Geophys Res (Space Phys) 115(A7): A07301. https://doi.org/10.1029/2009JA015154.
Santarelli L, Palangio P, De Lauretis M. 2014. Electromagnetic background noise at L'Aquila geomagnetic observatory. Ann Geophys 57(2): 0211. https://doi.org/10.4401/ag-6299.

Stokes AJ. 1989. A VLF receiver system for monitoring solar flares. J Am Assoc Var Star Obs 18(2): 146-147. Available at https://ui. adsabs.harvard.edu/abs/1989JAVSO.18.146S.

Thomson NR, Rodger CJ, Clilverd MA. 2005. Large solar flares and their ionospheric D region enhancements. J Geophys Res (Space Phys) 110(A6): A06306. https://doi.org/10.1029/2005JA011008.

Wenzel D, Jakowski N, Berdermann J, Mayer C, Valladares C, Heber B. 2016. Global ionospheric flare detection system (GIFDS). J Atmos Sol-Terr Phys 138: 233-242. https://doi.org/ 10.1016/j.jastp.2015.12.011.

Cite this article as: Guerrero A, Cid C, García A, Domínguez E, Montoya F, et al. 2021. The space weather station at the University of Alcala. J. Space Weather Space Clim. 11, 24. https://doi.org/10.1051/swsc/2021007. 